\title{
Apparent Diffusion Coefficient Mapping Using a Multi-Shot Spiral MRI Sequence of the Rat Brain
}

\author{
Tomokazu Numano ${ }^{1,2^{*}}$, Koji Hyodo², Naotaka Nitta², Junichi Hata ${ }^{3}$, Nobuaki Iwasaki ${ }^{4}$, \\ Kazuhiro Homma ${ }^{2}$ \\ ${ }^{1}$ Department of Radiological Science, Tokyo Metropolitan University, Tokyo, Japan \\ ${ }^{2}$ National Institute of Advanced Industrial Science and Technology, Tsukuba, Japan \\ ${ }^{3}$ Department of Radiology, The University of Tokyo Hospital, Tokyo, Japan \\ ${ }^{4}$ Department of Pediatrics, Ibaraki Prefectural University of Health Sciences, Ibaraki, Japan \\ Email: ${ }^{*}$-numano@tmu.ac.jp
}

Received 15 November 2013; revised 15 December 2013; accepted 25 December 2013

Copyright (C) 2014 by authors and Scientific Research Publishing Inc.

This work is licensed under the Creative Commons Attribution International License (CC BY). http://creativecommons.org/licenses/by/4.0/ (c) (i) Open Access

\begin{abstract}
Purpose: Commonly used diffusion weighted (DW) imaging such as DW spin echo (SE) type echo planar imaging (DW-SE-EPI) is known to be a snapshot-like acquisition and to have a relatively high signal-to-noise ratio. Spiral MRI sequence (SPIRAL) has characteristics similar to these of EPI, but it has rarely been used for diffusion-weighted imaging (DWI). In vivo DW-SPIRAL of the rat brain has almost never been reported. Our purpose in this study was to examine the potential of SE-type two-dimensional (2D) multi-shot spiral acquisition MRI for apparent diffusion coefficient (ADC) mapping of the rat brain in vivo. Materials and Methods: We made an SE-type DW-2D-spiral MRI sequence (DW-SPIRAL) which was prepared on a 2.0-T animal-experiment MR scanner. Comparing the phantom experimental result of DW-SPIRAL with the phantom experimental result of DW SE-type echo-planar imaging (DW-SE-EPI) and conventional DW spin echo imaging (DW-SE), we estimated the characteristics of DW-SPIRAL and assessed the clinical application of DW-SPIRAL in an animal experiment on the rat brain. Results: There was not much difference between the calculated water/glycerol phantom diffusion coefficient of DW-SPIRAL and the calculated diffusion coefficient of DW-SE. This result shows that the DW-SPIRAL sequence is appropriate for use in diffusion weighted imaging. There were fewer phantom image distortions and ghosting artifacts with DW-SPIRAL than with DW-SE-EPI, and this tendency was similar in the animal experiment on the rat brain. Conclusion: The DW-SPIRAL sequence had been successfully tested in phantom experiments and rat brain experiments. It has been demonstrated that the DW-SPIRAL sequence is capable of producing in vivo rat brain DWI.
\end{abstract}

${ }^{*}$ Corresponding author. 


\section{Keywords}

\section{Diffusion Weighted Imaging (DWI); Echo Planner Imaging (EPI); Spiral MRI Sequence; Apparent Diffusion Coefficient (ADC)}

\section{Introduction}

A spiral MRI sequence (SPIRAL) allows fast scanning with a relatively high signal-to-noise ratio (SNR), and it is relatively insensitive to motion [1]-[3]. Due to the spiral design of the k-space trajectory (gradient waveforms), middle and low-level MRI scanners do not allow straightforward implementation of SPIRAL. In particular, a single-shot spiral scan places high demands on gradient hardware, requiring careful design of the gradient waveforms. One hardware limitation of some MRI scanners is the ramping duty cycle. This duty cycle limit is related to the percentage of scan time spent on ramping (altering) the gradient amplitude, and stems from restrictions on the amount of heat dissipated by the pulse-width modulator that controls the output voltage of the gradient amplifier. This type of gradient amplifier limitation is particularly restrictive for spiral scans in which the gradient amplitude changes continuously during data collection. Furthermore, because the image reconstruction of SPIRAL is complicated, it is not used much. The Cartesian coordinates of the k-space points sampled along spiral trajectories do not form a uniform grid; thus, the fast Fourier transformation algorithm cannot be applied in the conventional manner for image reconstruction [1]-[4].

A spin-echo-type echo-planar imaging (SE-EPI) module is often used for diffusion-weighted imaging (DWI) because of its snapshot-like acquisition and relatively high SNR [5], [6]. Diffusion weighted SE-EPI (DW-SE-EPI) has become an important clinical modality for early detection of stroke [5], [7]. In addition, separation and extraction of white matter and gray matter [8], [9], and brain fibers related to anisotropy in water diffusion were investigated by using DW-SE-EPI with diffusion tensor magnetic resonance imaging (DTI) [6], [10]-[12]. Although SE-EPI is a mainstream DWI pulse sequence, one of its weak points is that a relatively short echo time (TE) is difficult to achieve. In contrast to EPI, the SPIRAL sequence k-space trajectory takes the form of a spiral. This has an advantage in that low spatial-frequency components of the k-space are acquired first, so the effective TE is short. Moreover, SPIRAL has other advantages that are related to short echo times. Very fast imaging of EPI needs rapid gradient switching. Each frequency-encoded gradient lobe is sampled rapidly with very high gradient amplitude, resulting in a very large signal bandwidth. This rather slow sampling rate means that the signal bandwidth in the phase-encode direction may be as little as $10 \mathrm{~Hz}$ per pixel. In particular, air-tissue boundaries result in large image distortions, and these become more severe at higher magnetic field strengths. Because SPIRAL does not need rectangular fast gradient switching for EPI encoding, the image distortion of SPIRAL is not severe. SPIRAL is able to reduce image distortions and it is predictably effective for animal experiments with a small field of view (FOV). Some studies have recently explored the spiral readout trajectory in DWI or DTI [13]-[17]. However, in animal experiments, the number of spiral readout trajectories in DWI or DTI has decreased markedly [18]. In rat experiments, spiral readout imaging has been rarely used for DWI, DTI, or conventional (T1, or T2-weighted) imaging.

In this study, we demonstrated an apparent diffusion coefficient map (ADC map) from the in vivo rat brain with a 2.0-T animal experiment MR imager by using an SE-type diffusion-weighted (DW) two-dimensional multi-shot spiral acquisition (DW-SPIRAL) which we developed. Basically, the SPIRAL technique offers a clear advantage over the EPI technique whenever a short TE and increased robustness to image distortions are of interest, as is typically the case with DWI.

\section{Materials and Methods}

\subsection{DW-SPIRAL Sequence Implementation and Optimization}

Figure 1 shows the timing chart of the self-made SE-type DW two-dimensional multi-shot spiral acquisition (DW-SPIRAL). This sequence combines motion proving gradients (MPG) with a conventional spiral imaging module. The MPG was applied symmetrically around a refocusing (180-degree RF) pulse, and it could be applied in any direction. Like EPI, SPIRAL is a very fast MRI technique capable of efficiently sampling the entire 


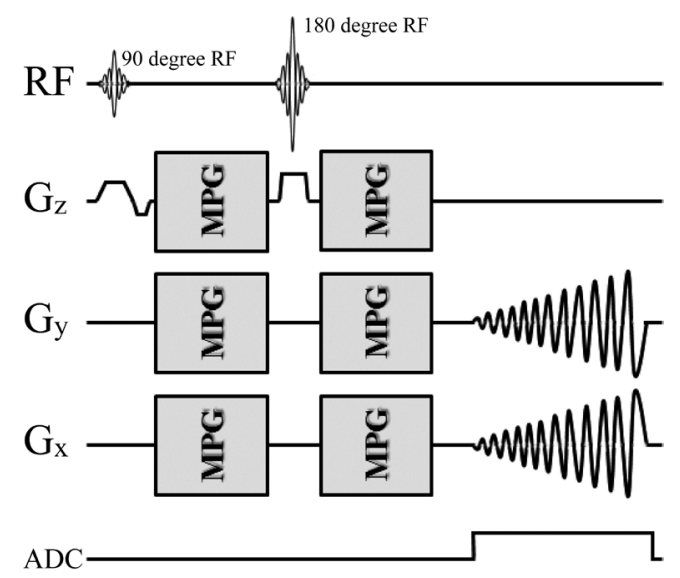

Figure 1. Diagram of the SE-type 2D spiral MR pulse sequence modified for diffusion imaging (DW-SPIRAL). The motion proving gradients (MPG) were applied symmetrically around a refocusing (180 degree RF) pulse, which could be applied in any direction. In SPIRAL imaging, $G_{x}$ and $G_{v}$ could handle the readout-encode and phase-encode simultaneously, as well as act independently of each other. SPIRAL imaging has the advantage that the low k-space spatial frequencies (center of the $\mathrm{k}$-space) are acquired first, and thus the effective TE is short.

k-space of interest in just one segment (single shot) or in a few k-space segments (multi-shot). All MRI experiments were performed on a 2.0-T Biospec 20/30 System with a B-GA20 Gradient System (BRUKER, Karlsruhe, Germany) that had a maximum gradient strength of $100 \mathrm{mT} / \mathrm{m}$. The MR data acquisition, image reconstruction, and MR pulse sequence development were performed with a ParaVision ver.4 (BRUKER) software system. The data re-gridding (conversion from spiral to Cartesian k-space), and image reconstruction used a full production copy of spiral image reconstruction (BRUKER). A 70-mm-ID birdcage coil (BRUKER) tuned to $85 \mathrm{MHz}$ for proton resonance was used for phantom experiments.

To verify the implementation of the DW-SPIRAL sequence, we monitored its gradient waveform with a self-made monitoring system (LabVIEW, National Instruments, TX, USA). Figure 2 shows the measured gradient waveforms of the DW-SPIRAL sequence (Figure 2(a)) and the measured gradient waveforms of the DWSE-EPI sequence (Figure 2(b)), using the self-made monitoring system. These have the same FOV, matrix, segments (8 shot), MPG power, MPG duration $(\delta)$, and MPG distance $(\Delta)$. These gradient waveforms verified that the DW-SPIRAL sequence was mounted in the MRI and that it was operating. With these imaging parameters, because the sequential (high-low-high) k-space acquisition-order EPI takes a little time to elapse before the middle of the k-space, the TE of the DW-SPIRAL was shorter than the TE of DW-SE-EPI without TE shortening techniques (such as Parallel imaging etc.).

To optimize the imaging parameters (especially the number of shots) of the DW-SPIRAL, we experimented with different numbers for shot imaging (image data not shown). The final imaging parameter was determined based on the results of these phantom experiments. Reasonable imaging parameters were an FOV of $80 \mathrm{~mm}$, an image matrix of 128, and a shot of 8 (segment of 8).

\subsection{Verification Experiments for the Diffusion Effect of DW-SPIRAL}

The effect of diffusion on the contrast of DW-SPIRAL was verified by comparing the water phantom diffusion coefficient and water solution of glycerol phantom diffusion coefficient with conventional spin echo DWI (SE-DWI) and DW-SE-EPI. The phantom was made of a series of 1.5-cm-diameter tubes containing various concentrations of glycerol. The concentration of glycerol varied from $0 \mathrm{wt} \%$ (water phantom) to $72 \mathrm{wt} \%$, with an arbitrary step. T1 of the phantom measured with a short-TE spin-echo sequence with different TR values (saturation recovery method). T2 of the phantom was measured with a long-TR spin-echo sequence with different TE values (multi echo method). The instrumental T1/T2 values of the water phantom, $15 \mathrm{wt} \%$ phantom, $30 \mathrm{wt} \%$ phantom, 39 wt\% phantom, 56 wt\% phantom, and 72 wt\% phantom were 2476 ms/907 ms, 1942 ms/150 ms, $1777 \mathrm{~ms} / 120 \mathrm{~ms}, 1074 \mathrm{~ms} / 118 \mathrm{~ms}, 658 \mathrm{~ms} / 147 \mathrm{~ms}$, and $349 \mathrm{~ms} / 123 \mathrm{~ms}$, respectively. The SE-DWI coefficient is a standard value diffusion coefficient in this MRI system. The imaging parameters of DW-SPIRAL were 3000 $\mathrm{ms} / 37.5 \mathrm{~ms}$ (TR/TE), slice thickness of $3 \mathrm{~mm}$, MPG duration $(\delta)$ of $13 \mathrm{~ms}$, MPG separation $(\Delta)$ of $18 \mathrm{~ms}$, 

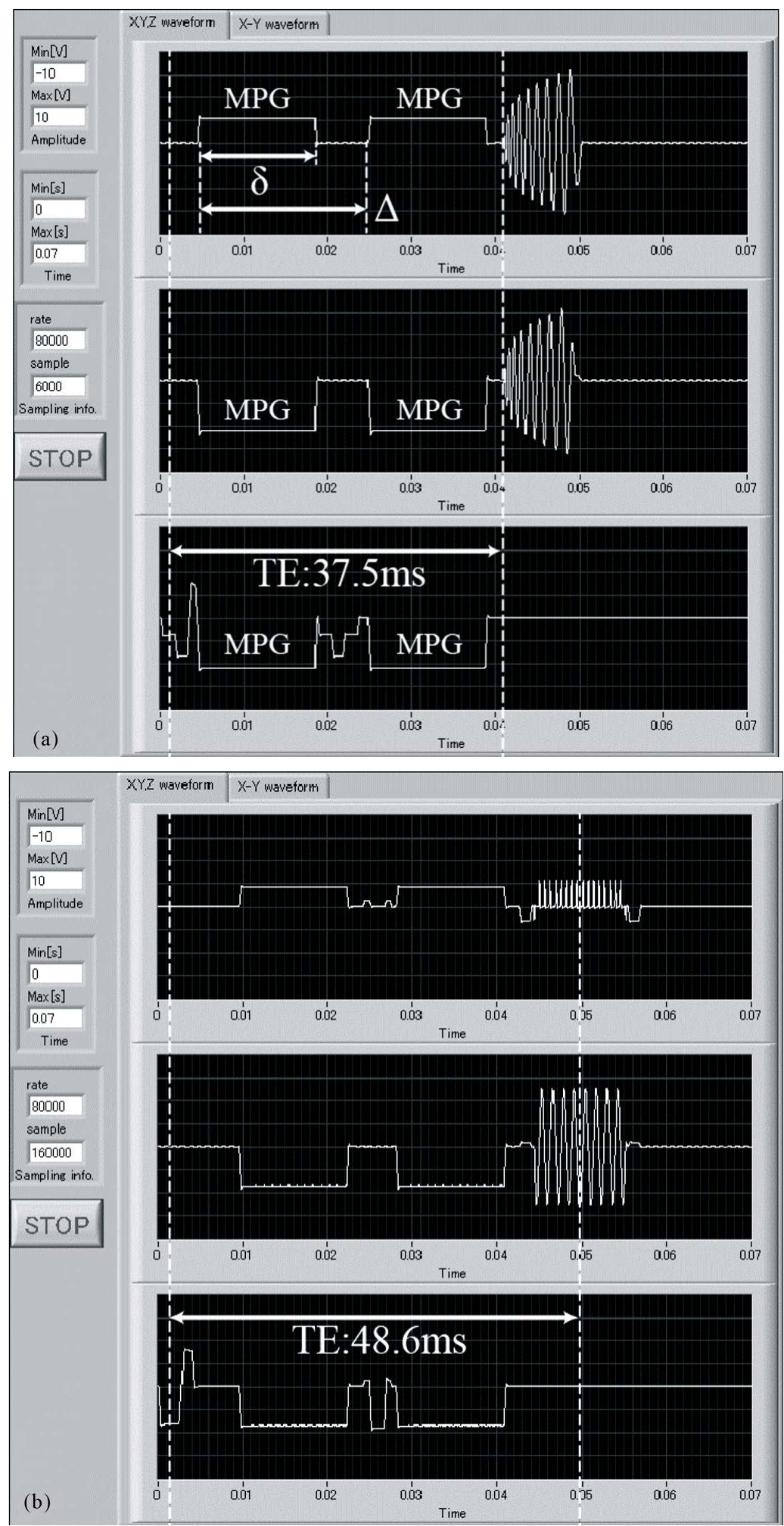

Figure 2. The DW-SPIRAL sequence gradient waveforms (a) and the DW-SE-EPI sequence gradient waveforms (b). They have the same FOV, matrix, segment (8 shot), MPG power, MPG duration $(\delta)$, and MPG distance $(\Delta)$. For these imaging parameters, because the sequential (high-low-high) k-space acquisition-order EPI engages a time that elapses before the middle of the k-space, the TE of DW-SPIRAL was shorter than the TE of DW-SE-EPI without TE shortening techniques (such as Parallel imaging etc.). 
b-value of $0,330,648,1194 \mathrm{~s} / \mathrm{mm}^{2}$, matrix of $64, \mathrm{FOV}$ of $80 \mathrm{~mm}$, and shot of 8 . The total acquisition time was $1 \mathrm{~min} 36 \mathrm{~s}$. The imaging parameters of SE-DWI were $3000 \mathrm{~ms} / 37 \mathrm{~ms}$ (TR/TE), slice thickness of $3 \mathrm{~mm}$, MPG duration $(\delta)$ of $13 \mathrm{~ms}$, MPG separation $(\Delta)$ of $18 \mathrm{~ms}$, b-value of $0,330,648,1194 \mathrm{~s} / \mathrm{mm}^{2}$, matrix of $64 \times 64$, and FOV of $80 \times 80 \mathrm{~mm}$. The total acquisition time was $12 \mathrm{~min} 38 \mathrm{~s}$. The imaging parameters of DW-SE-EPI were $3000 \mathrm{~ms} / 48.6 \mathrm{~ms}$ (TR/TE), slice thickness of $3 \mathrm{~mm}$, MPG duration $(\delta)$ of $13 \mathrm{~ms}$, MPG separation $(\Delta)$ of $18 \mathrm{~ms}$, b-value of $0,330,648,1194 \mathrm{~s} / \mathrm{mm}^{2}$, matrix of 64 , FOV of $80 \mathrm{~mm}$, and segment of 8 . The total acquisition time was $1 \mathrm{~min} 36 \mathrm{~s}$. Because the water diffusion in the phantom was isotropic (self-diffusion), MPG was applied to only the $\mathrm{X}$-axis in each sequence. Each five-image set was then used to produce a pixel-by-pixel parametric image of the ADC map.

\subsection{Verification Experiment for Image Distortion and Spatial Resolution of DW-SPIRAL}

The image distortion of T2-weighted DW-SPIRAL (b-value, $0 \mathrm{~s} / \mathrm{mm}^{2}$; SPIRAL) was verified by comparing each image of the accuracy evaluation phantom with T2-weighted spin echo (SE), T2-weighted fast spin echo (Fast $\mathrm{SE}$ ), and T2-weighted DW-SE-EPI (b-value, $0 \mathrm{~s} / \mathrm{mm}^{2}$; SE-EPI). The phantom was imaged with each sequence; the phantom image of SE was used as a benchmark, while the image distortion was assessed by comparing the vertical, horizontal, and diagonal diameter (Figure 3(a)). The frequency encoding direction of Fast SE and SE-EPI was horizontal. The spatial resolution of SPIRAL was evaluated using a self-made spatial resolution evaluation phantom (Figure 3(b)). This phantom was made from a glass capillary enclosed within a plastic tube. The spatial resolution of SPIRAL was verified by comparing the normalized signal intensity profiles across the images in Figure 4(a) at the locations indicated by the white lines. The imaging parameters of DW-SPIRAL were $3000 \mathrm{~ms} / 37.5 \mathrm{~ms}$ (TR/TE), slice thickness of $2 \mathrm{~mm}$, matrix of 128, FOV of $80 \mathrm{~mm}$, and shot of 8 . The total acquisition time was $24 \mathrm{~s}$. The imaging parameters of SE were $3000 \mathrm{~ms} / 37 \mathrm{~ms}$ (TR/TE), slice thickness of $2 \mathrm{~mm}$, matrix of $128 \times 128$, and FOV of $80 \times 80 \mathrm{~mm}$. The total acquisition time was $7 \mathrm{~m} 24 \mathrm{~s}$. The imaging parameters of Fast SE were $3000 \mathrm{~ms} / 100 \mathrm{~ms}$ (TR/ effective TE), slice thickness of $2 \mathrm{~mm}$, matrix of $128 \times 128$, FOV of $80 \times$ $80 \mathrm{~mm}$, and echo train length (ETL) of 16. The total acquisition time was $24 \mathrm{~s}$. The imaging parameters of SE-EPI were $3000 \mathrm{~ms} / 48.6 \mathrm{~ms}$ (TR/ effective TE), slice thickness of $2 \mathrm{~mm}$, matrix of $128 \times 128$, FOV of $80 \times$ $80 \mathrm{~mm}$, and segment of 8 . The total acquisition time was $24 \mathrm{~s}$.

\subsection{DW-SPIRAL in Vivo Experiment}

For the in vivo study, male Crj:Wistar rats (450 g) were anesthetized by intraperitoneal injection of ketamine $100 \mathrm{mg} / \mathrm{kg}$ body weight (Kyoritsu Seiyaku Co., Tokyo, Japan), xylazine $10 \mathrm{mg} / \mathrm{kg}$ body weight (Bayer Holding Ltd., Tokyo, Japan), and atropine $0.05 \mathrm{mg} / \mathrm{kg}$ body weight (Terumo Co., Tokyo, Japan). Each anesthetized rat was placed on an acrylic bed and subjected to scanning. Additionally, anesthesia was maintained using isoflurane (1.5\%) inhalation anesthesia. This study was performed in accordance with animal protection laws and approved by the Animal Ethical Committee of the National Institute of Advanced Industrial Science and Technol-

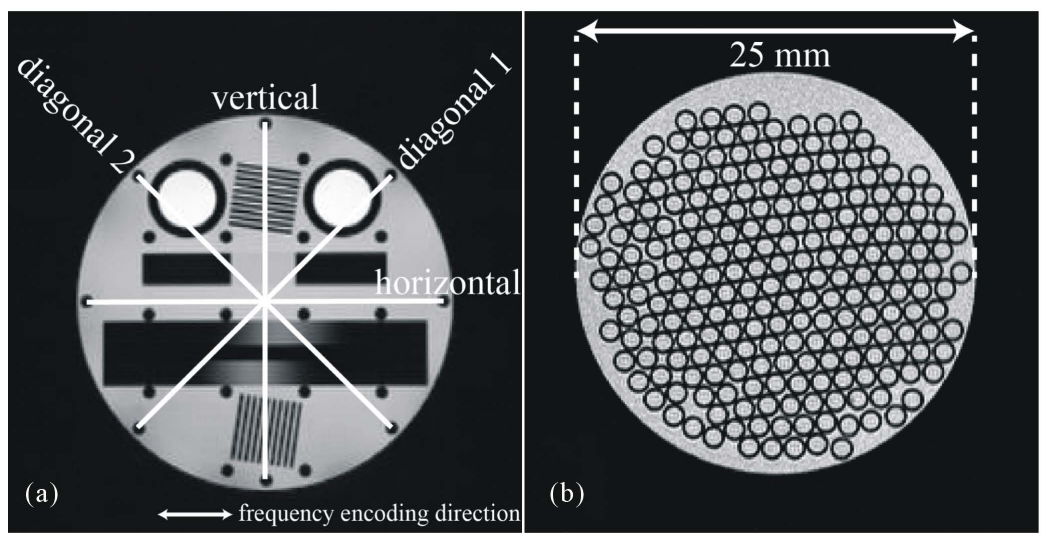

Figure 3. An accuracy evaluation phantom image and the measurement distance point (a). The frequency encoding direction of Fast SE and SE-EPI was horizontal. A self-made spatial resolution evaluation phantom was made from a glass capillary enclosed within a plastic tube (b). 


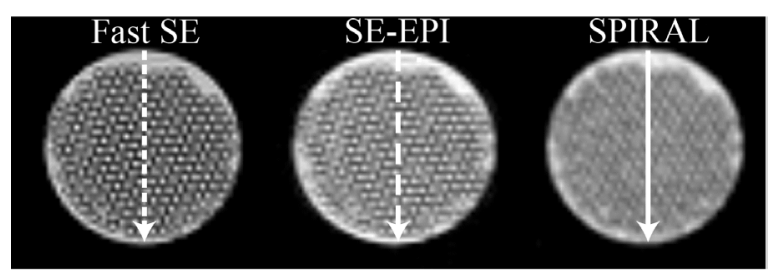

(a)

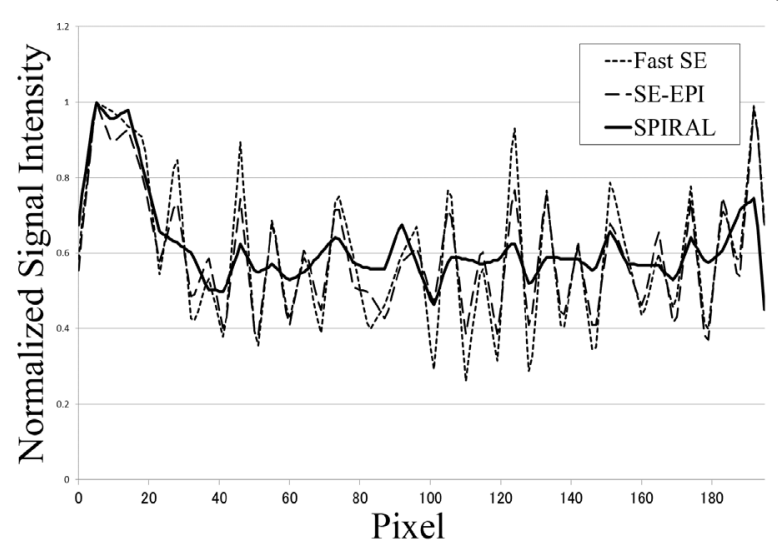

(b)

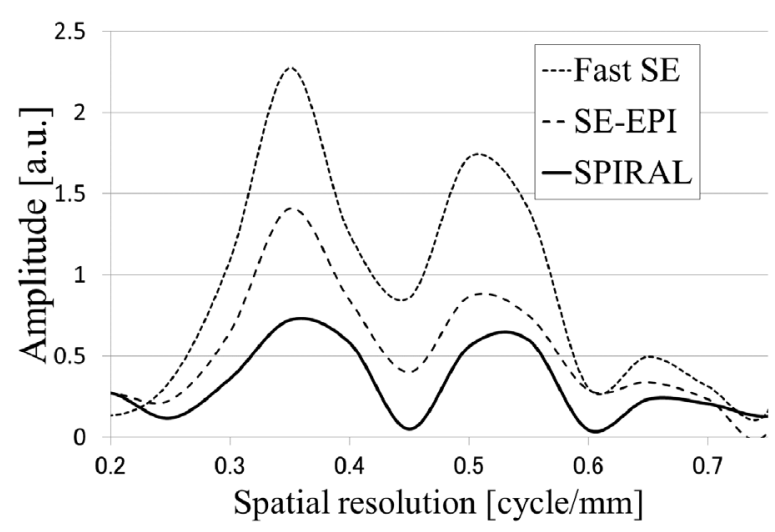

(c)

Figure 4. Self-made spatial resolution evaluation phantom images of each sequence and position of the profile line (a). The pixel size of each MR image was $625 \mu \mathrm{m}$. This pixel size was determined by the SPIRAL sequence limitation. Image profile curves of the self-made spatial resolution evaluation phantom (b). The image profile curve of Fast SE (dashed line) was used as an ideal spatial resolution. The profile curve of SE-EPI (large-spaced dashed line) was in close agreement with the profile curve of Fast SE. The profile curve of SPIRAL (solid line) was not in agreement (the SPIRAL profile showed a mild shift) with the profile curve of Fast SE. Fourier analysis of phantom image profile curves (c). This result shows that the spatial resolution of SPIRAL was inferior to the spatial resolution of SE-EPI.

ogy. A 45-mm-ID saddle coil (Takashima Seisakusho, Hino, Japan) tuned to $85 \mathrm{MHz}$ for proton resonance was used for rat brain experiments. The clinical application of DW-SPIRAL was verified by comparison of the rat diffusion weighted images and ADC-map with DW-SE-EPI. To allow for anatomic references, T2-weighted Fast SE of the rat brain was performed. The imaging parameters of DW-SPIRAL were $5000 \mathrm{~ms} / 37.5 \mathrm{~ms}$ (TR/TE), slice thickness of $2 \mathrm{~mm}$, MPG duration $(\delta)$ of $13 \mathrm{~ms}$, MPG separation $(\Delta)$ of $18 \mathrm{~ms}$, b-value of 0 , 330, $648,1194 \mathrm{~s} / \mathrm{mm}^{2}$, matrix of 128 , FOV of $80 \mathrm{~mm}$, and shot of 8 . The total acquisition time was $2 \mathrm{~min} 40 \mathrm{~s}$. The imaging parameters of DW-SE-EPI were $5000 \mathrm{~ms} / 48.6 \mathrm{~ms}$ (TR/ effective TE), slice thickness of $2 \mathrm{~mm}$, MPG duration $(\delta)$ of $13 \mathrm{~ms}$, MPG separation $(\Delta)$ of $18 \mathrm{~ms}$, b-value of $0,330,648,1194 \mathrm{~s} / \mathrm{mm}^{2}$, matrix of $128 \times 128$, FOV of $80 \times 80 \mathrm{~mm}$, and segment of 8 . The total acquisition time was 2 min $40 \mathrm{~s}$. The imaging parameters of Fast SE were $5000 \mathrm{~ms} / 54 \mathrm{~ms}$ (TR/effective TE), slice thickness of $2 \mathrm{~mm}$, matrix of $256 \times 256$ (192), FOV of 60 $\times 60 \mathrm{~mm}$, and ETL of 8 . The total acquisition time was $4 \mathrm{~min}$. For this study, to confirm the efficacy of diffusion image contrast with DW-SPIRAL, MPG was applied only to the X-axis.

\section{Results}

\subsection{Diffusion Effect}

The measured diffusion coefficients of the water phantoms and water solution of glycerol phantoms by each MR pulse sequence are listed in Table 1 . These measurements were repeating three times for each MR pulse sequence. The room temperature of the MR magnet room was maintained at 20 degrees Celsius during the experiments. In all sequences, the repetition time (TR) was longer than the T1 of phantoms; thus, the effects of T1 relaxation were minimal. According to published data, the diffusion coefficient of water at 20 degrees Celsius is $2.023 \times 10^{-3}$ $\mathrm{mm}^{2} / \mathrm{s}$. The diffusion coefficient for the SE-DWI was in agreement with this value. Therefore, the diffusion coefficient for the SE-DWI was taken as having an ideal value in this experiment. The diffusion coefficient for the 
Table 1. Diffusion coefficient in the water/glycerol phantoms obtained with different MR pulse sequences.

\begin{tabular}{ccccccc}
\hline & \multirow{2}{*}{ Water } & \multicolumn{5}{c}{ Water solution of glycerol } \\
\cline { 3 - 7 } & & $15[\mathrm{wt} \%]$ & $30[\mathrm{wt} \%]$ & $39[\mathrm{wt} \%]$ & $56[\mathrm{wt} \%]$ & $72[\mathrm{wt} \%]$ \\
\hline Literature data & $2.023^{*}$ & - & - & - & - \\
SE-DWI & $1.979 \pm 0.016$ & $1.571 \pm 0.003$ & $1.074 \pm 0.002$ & $0.633 \pm 0.006$ & $0.302 \pm 0.007$ & $0.100 \pm 0.006$ \\
DW-SPIRAL & $1.801 \pm 0.055$ & $1.456 \pm 0.029$ & $1.115 \pm 0.032$ & $0.889 \pm 0.065$ & $0.377 \pm 0.057$ & $0.165 \pm 0.037$ \\
DW-SE-EPI & $1.939 \pm 0.055$ & $1.610 \pm 0.019$ & $1.074 \pm 0.009$ & $0.795 \pm 0.008$ & $0.371 \pm 0.010$ & $0.185 \pm 0.016$ \\
& \multicolumn{7}{c}{ Mean $\pm \mathrm{SD} \times 10^{-3}\left[\mathrm{~mm}^{2} / \mathrm{s}\right]$} \\
\hline
\end{tabular}

*Data courtesy of Dr.M.Holz, Institute of Phys. Chem, University of Karlsruhe, FRG.

DW-SPIRAL was $1.801 \times 10^{-3} \mathrm{~mm}^{2} / \mathrm{s}$. This result shows that the DW-SPIRAL sequence has a diffusion effect on DW-SPIRAL images. To evaluate the diffusion image contrast effect of DW-SPIRAL, the diffusion coefficient by SE-DWI $\left(\boldsymbol{D}_{\mathrm{SE}}\right)$ changes vs. the diffusion coefficient by DW-SPIRAL $\left(\boldsymbol{D}_{\text {SPIRAL }}\right)$, and diffusion coefficient by DW-SE-EPI ( $\left.\boldsymbol{D}_{\mathrm{EPI}}\right)$ were plotted (Figure 5$)$. The solid line shows linear regression of $\boldsymbol{D}_{\text {SPIRAL }}\left(\boldsymbol{D}_{\text {SPIRAL }}=0.84 \boldsymbol{D}_{\text {SE }}\right.$ $+0.18)$. The dashed line shows linear regression of $\boldsymbol{D}_{\mathrm{EPI}}\left(\boldsymbol{D}_{\mathrm{EPI}}=0.93 \boldsymbol{D}_{\mathrm{SE}}+0.12\right)$. If the diffusion coefficient by SE-DWI is equal to the diffusion coefficient by each DW sequence, the slope of the linear regression is 1 . Because the $\boldsymbol{D}_{\text {EPI }}$ slope is 0.93 , the DW-SE-EPI was almost equal to the contrast of SE-DWI. On the other hand, because the $\boldsymbol{D}_{\text {SPIRAL }}$ slope was 0.84, DW-SPIRAL was inferior to DW-SE-EPI in terms of the diffusion image contrast effect.

\subsection{Image Distortion and Spatial Resolution}

The phantom image size ratios between each sequence and the SE-sequence are listed in Table 2. In all phantom directions, the phantom image of Fast SE showed the least image distortion. In general, a single-shot (single-segment) EPI is extremely sensitive to image artifacts and distortion. The advantage of a multi-shot (multi-segment) EPI is that susceptibility effects can be reduced as the phase-encode bandwidth is effectively higher than for an equivalent single shot. One positive aspect here was that the image distortion of SE-EPI (8 segment) did not have any image distortions caused by susceptibility effects. In this experiment, the phantom image of SE-EPI showed slightly image distortion. In particular, the image distortion (expansion) in the horizontal direction was conspicuous. The SPIRAL image had lower distortion than the image distortion of SE-EPI.

Figure 4(b) shows the phantom image profile curve of each MR sequence. The phantom image profile of Fast SE was handled as a benchmark spatial resolution for this pixel size (this pixel size was determined by the SPIRAL sequence limitation). The profile curve of SE-EPI was in good agreement with the profile curve of Fast SE. In contrast, the profile curve of SPIRAL was not in agreement (the SPIRAL profile showed a mild shift) with the profile curve of Fast SE. Figure 4(c) shows Fourier analysis of phantom image profile curves. This result shows that the spatial resolution of SPIRAL was inferior to the spatial resolution of SE-EPI.

\subsection{Rat Experiments}

Figure 6 compares diffusion weighted images (b-value, 0 to $1194 \mathrm{~mm}^{2} / \mathrm{s}$ ) of axial slices with the DW-SPIRAL (top row) and DW-SE-EPI (bottom row) sequences. These images were keeping the image brightness constant. No image ghost occurred in the DW-SPIRAL sequence. On the other hand, the DW-SE-EPI sequence had frequent image ghosts with high b-values $\left(600 \mathrm{~mm}^{2} / \mathrm{s}\right.$ or more).

Figure 7(a) compares T2-weighted images (b-value, $0 \mathrm{~mm}^{2} / \mathrm{s}$ ) of two different slices obtained with the Fast SE (top row), DW-SE-EPI (middle row), and DW-SPIRAL (bottom row) sequences. The slightly higher signal intensity observed in DW-SPIRAL vs. DW-SE-EPI images could be the result of the shorter TE in the spiral acquisition (37.5 ms vs. $48.6 \mathrm{~ms}$ ) and of less signal loss due to T2* relaxation. As for both DW-SE-EPI slice images, image distortion (expansion) in the horizontal direction (readout direction) was seen, as in the phantom experiments. However, the spatial resolution of DW-SPIRAL was inferior to the spatial resolution of DW-SE-EPI. The DW-SPIRAL did not have enough spatial resolution, and could not image the corpus callosum. 
Table 2. Imaged phantom size ratio between each MR sequence and SE-sequence.

\begin{tabular}{rcccc}
\hline & Vertical & Horizontal & Diagonal 1 & Diagonal 2 \\
\hline Fast SE & $0.39 \%$ & $0.00 \%$ & $0.53 \%$ & $0.26 \%$ \\
SE-EPI & $0.76 \%$ & $2.63 \%$ & $0.84 \%$ & $0.82 \%$ \\
SPIRAL & $0.77 \%$ & $0.74 \%$ & $0.79 \%$ & $0.54 \%$ \\
\hline
\end{tabular}

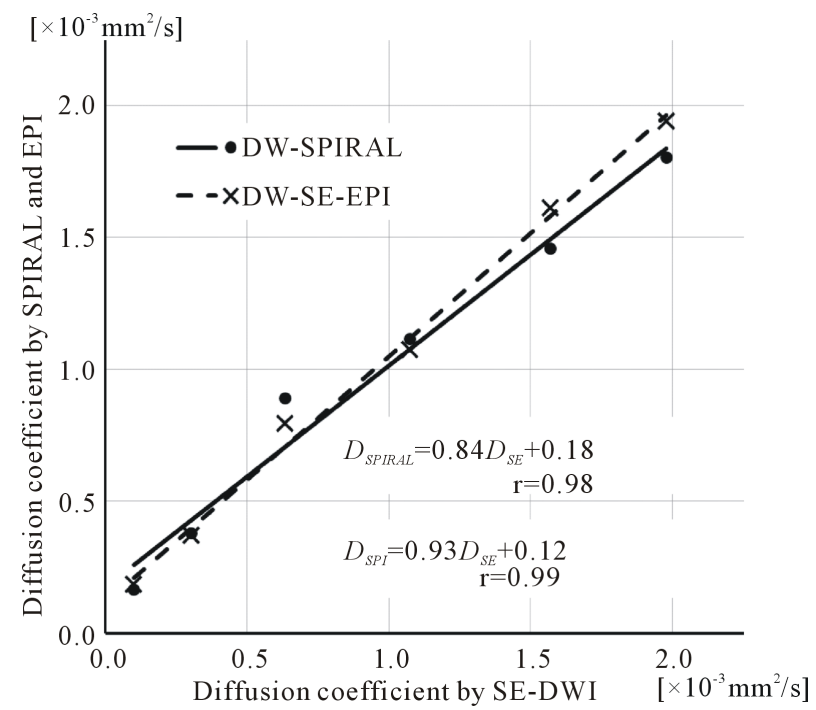

Figure 5. Phantom experiment results with different k-space acquisition orders for the water/glycerol phantoms. Plot of the diffusion coefficient by SE-DWI $\left(D_{S E}\right)$ changes vs. the diffusion coefficient by DW-SPIRAL $\left(D_{S P I R A L}\right)$, and diffusion coefficient by DW-SE-EPI $\left(D_{E P I}\right)$. The solid line is linear regression of $D_{\text {SPIRAL }}\left(D_{\text {SPIRAL }}=0.84 D_{S E}+0.18\right)$. The dashed line is linear regression of $D_{E P I}\left(D_{E P I}=0.93 D_{S E}+0.12\right)$.

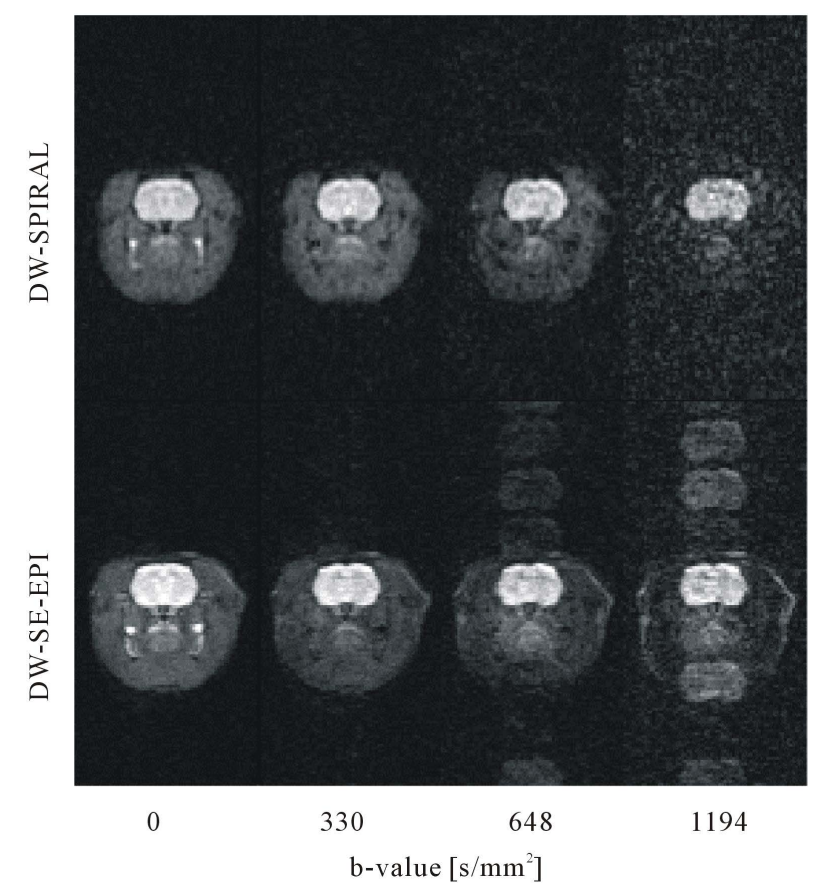

Figure 6. Comparison of diffusion-weighted images (b-value, 0 to $1194 \mathrm{~mm}^{2} / \mathrm{s}$ ) of axial slices with the DW-SPIRAL (top row) and DW-SE-EPI (bottom row) sequences. These images were with a maintained image brightness constant. The DW-SE-EPI sequence had image ghosts at high b-values $\left(600 \mathrm{~mm}^{2} / \mathrm{s}\right.$ or more). 

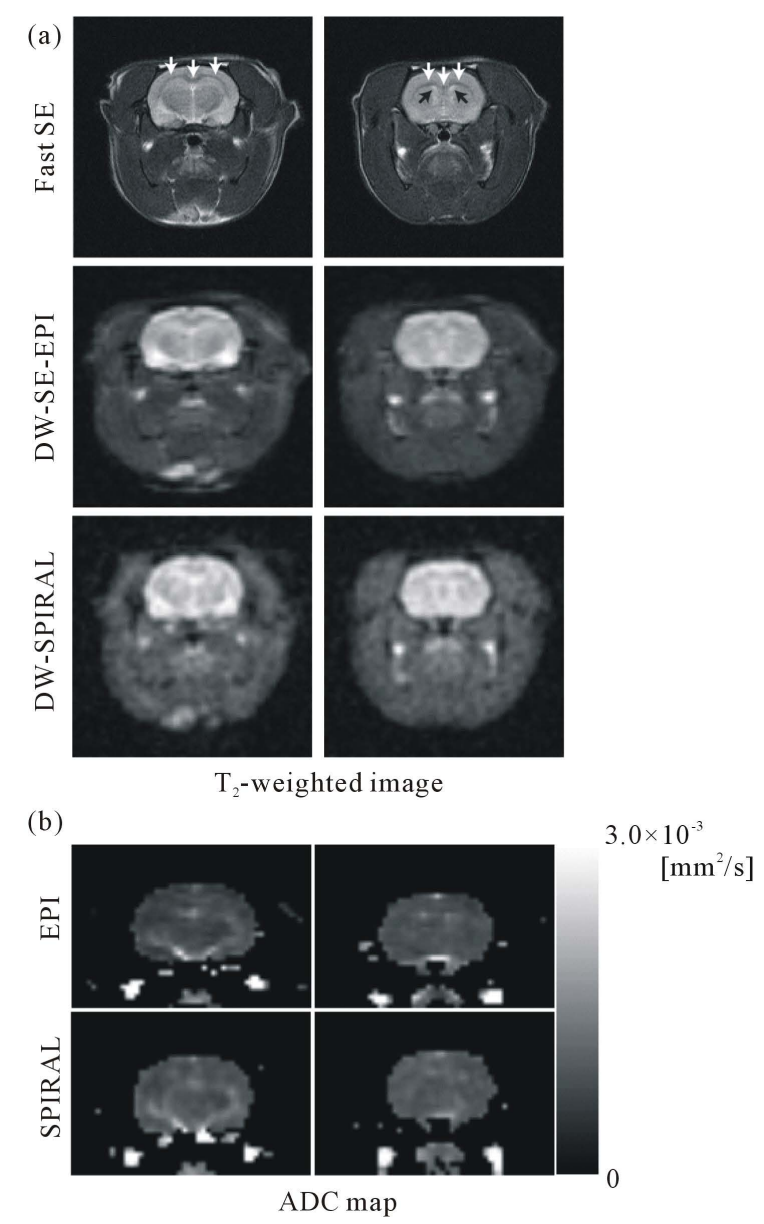

Figure 7. Comparison of T2-weighted images (b-value, $0 \mathrm{~mm}^{2} / \mathrm{s}$ ) of two different slices obtained with the Fast SE (top row), DW-SE-EPI (middle row), and DW-SPIRAL (bottom row) sequences (a). The white arrows show the corpus callosum, and the black arrows show the lateral ventricle. The pixel sizes of each MR image were $234 \mu \mathrm{m}$ (Fast SE) and $625 \mu \mathrm{m}$ (DW-SE-EPI, DW-SPIRAL). Comparison of ADC maps of two different slices obtained with the DW-SE-EPI (top row) and DW-SPIRAL (bottom row) sequences (b). These ADC maps displayed only the brain area.

Figure 7(b) compares ADC maps of two different slices obtained with DW-SE-EPI (top row) and DWSPIRAL (bottom row) sequences. These ADC maps were made using regional extraction of the brain area. The ADC map of DW-SE-EPI had enough spatial resolution to draw the lateral ventricle. On the other hand, because the DW-SPIRAL did not have spatial resolution, it was difficult to draw the lateral ventricle in the ADC-map.

\section{Discussion}

In this study, we demonstrated the feasibility of a combined 2D-spiral MRI sequence and diffusion-weighted imaging. Our spin echo (SE) type diffusion-weighted (DW) two-dimensional (2D) multi-shot spiral acquisition MRI (DW-SPIRAL) had diffusion contrast, and could create an ADC map of the rat brain. An animal experiment with MRI demands higher image resolution and it requires low image distortion and few image ghosts. The DW-SPIRAL sequence reduces most image distortions and image ghosts, which appear frequently in images obtained from EPI sequences. However, the spatial image resolution and the diffusion image contrast effect of DW-SPIRAL were inferior to the DW-SE-EPI. Features and limitations of the DW-SPIRAL are described below.

First, the spatial resolution of SPIRAL was inferior to the spatial resolution of SE-EPI (Figure 4). Adequate spatial resolution should be the overriding concern in medical imaging technology. In this respect, the SPIRAL sequence is inadequate for the small FOV in animal MRI studies. In spiral MRI, the observed signal represents 
2D Fourier transformation of the object along a spiral trajectory in k-space. The object image can be reconstructed from the spiral MRI signal by using various algorithms. The widely used Kaiser-Bessel gridding method speeds up the reconstruction by interpolating the data onto Cartesian coordinates and using a Kaiser-Bessel convolution kernel followed by a fast inverse Fourier transformation. However, the performance of the Kaiser-Bessel gridding method is highly dependent on the selection of the kernel function parameter; even by using the optimal parameter, the reconstructed image could still have trajectory-dependent local distortion (decreased spatial resolution) [4], [19]. In this study, we used a full production copy of spiral image reconstruction (BRUKER) with the Kaiser-Bessel gridding method. The DW-SPIRAL using the Kaiser-Bessel gridding method has low spatial resolution.

Second, the diffusion image contrast effect of the DW-SPIRAL was inferior to the DW-SE-EPI. In the SE-DWI, a conventional SE-sequence used only a pure spin echo signal, caused by the 180 degree refocusing RF pulse. For this reason, SE-DWI was more robust against factors such as susceptibility artifacts than DW-SPIRAL or DW-SE-EPI. This robustness helped the diffusion coefficient of SE-DWI to approach the published value and achieve a low standard deviation (SD) of the SE-DWI ADC values. Additionally, the ADC-map of SE-DWI had a lower image noise (low SD value). Therefore, the diffusion coefficient of SE-DWI is the standard value of a diffusion coefficient in this MRI system. The water diffusion coefficient showed a 9\% disparity between DW-SPIRAL and the "standard value" diffusion coefficient of SE-DWI. On the other hand, the difference between the water diffusion coefficient of SE-DWI and DW-SE-EPI was 2\%. From the experimental results using water/glycerol phantoms, the contrast of DW-SPIRAL was 0.9 times that of the contrast of DW-SE-EPI (Figure 5). The diffusion contrast effect of the DW-SPIRAL was inferior to DW-SE-EPI. We carried out the phantom experiments under the logic that because a phantom is stationary (does not have pulsation or respiration), the motion-induced phase errors with each SPIRAL shot do not have much influence on the diffusion contrast effect. Probably, the low signal-to-noise ratio MR signal at a high b-value acquisition influenced the SPIRAL re-gridding method, reducing the dynamic range of the diffusion-weighted effect. The decrease in diffusion contrast was likely caused by the particular SPIRAL re-gridding method. The DW-SPIRAL had a disadvantage not only in terms of spatial resolution, but also regarding the diffusion contrast. Although we only investigated a self-made DW-SPIRAL sequence, neither image ghosts nor image distortion occurred in the DW-SPIRAL sequence. Even if the DW-SE-EPI has higher spatial resolution compared with DW-SPIRAL, the image ghosts of DW-SE-EPI might spoil the spatial resolution.

Although it might not be a serious problem, the major limitation of these experiments was the lack of a sufficient SNR for all DWI images (Figures 6 and 7). The magnet strength of $2.0 \mathrm{~T}$ was not good enough for the rat brain DWI. To increase the SNR, number averaging can be used; however, this generally leads to view-to-view phase variations caused by motion while the MPG is turned on. Body motion under light anesthesia, pulsation of blood, or pulsation of cerebrospinal fluid (CSF), for example, can cause these variations. One approach to correcting these variations is to acquire additional navigator echo data that can be used to resolve the phase error. Another remedy is an MR sequence synchronized to a heart rate monitor (pulse oximeter). This technique avoids the phase error of cerebrospinal fluid (CSF) and blood pulsation. As stated above, image quality improvement cannot be expected with simple averaging. Also, because the DW-SPIRAL used a multi-shot technique, it is preferable that the additional navigator echo is acquired between each shot, although with a longer scan time requirement. Most recently, there was a report of inherent correction of motion-induced phase errors in multishot spiral DTI [20]. This method relies on sensitivity encoding to estimate the motion-induced phase error for each shot and subsequently use either a direct phase subtraction or an iterative conjugate gradient algorithm to correct for the resulting artifacts. The most important benefit of this method is that it effectively corrects for phase errors, signal loss, and aliasing artifacts caused by both body-rigid and nonrigid motion in multishot spiral DTI, without increasing the scan time. This method is vital to enhancing the image quality of DW-SPIRAL in vivo.

Spin-echo-type echo-planar imaging modules are the mainstream of DWI, combining TE shortening techniques such as Half Fourier imaging, Scan percentage, and Parallel imaging. These techniques contribute to effective TE shortening, so that the effective TE of the existing spin-echo-type EPI sequence is reasonable. In contrast, the spin-echo-type DW-SPIRAL sequence can essentially shorten the effective TE, and it does not require any TE shortening techniques. With this research, however, the DW-SPIRAL sequence with the Kaiser-Bessel gridding method showed insufficient spatial resolution of rat brain DWI. At some time in the future, DW-SE-EPI sequences combined with some effective TE shortening techniques will be the mainstream of DWI. 
Having said that, a compressed sensing reconstruction has been reported to improve the sensitivity of functional MRI by using a spiral sequence [21]. With the advent of a well-made SPIRAL re-gridding method, DWSPIRAL could become the mainstream of DWI.

\section{Conclusion}

In conclusion, a self-made SE-type DW-2D-spiral MRI sequence (DW-SPIRAL) was tested and discussed. From the results of phantom experiments, the DW-SPIRAL sequence produced diffusion-weighted contrast, although the spatial resolution was inferior to the SE-type EPI sequence. Judging from the results of animal experiments, the DW-SPIRAL sequence could be used to perform in vivo diffusion weighted imaging of the rat brain without being susceptible to distortion.

\section{Conflict of Interest}

This work supported in part by a Grant-in-Aid for Scientific Research (22791211, 23500961, 25461838).

\section{References}

[1] Papadakis, N.G., Wilkinson, A.A., Carpenter, T.A. and Hall, L.D., (1997) A General Method for Measurement of the Time Integral of Variant Magnetic Field Gradients: Application to 2D Spiral Imaging. Magnetic Resonance Imaging, 15, 567-578. http://dx.doi.org/10.1016/S0730-725X(97)00014-3

[2] Qian, Y., Zhao, T., Hue, Y.K., Ibrahim, T.S. and Boada, F.E. (2010) High-Resolution Spiral Imaging on a Whole-Body 7T Scanner with Minimized Image Blurring. Magnetic Resonance in Medicine, 63, 543-552. http://dx.doi.org/10.1002/mrm.22215

[3] Wu, B., Li, W., Avram, A.V., Gho, S.M. and Liu, C. (2010) Fast and Tissue-Optimized Mapping of Magnetic Susceptibility and T2* with Multi-Echo and Multi-Shot Spirals. NeuroImage, 59, 297-305. http://dx.doi.org/10.1016/j.neuroimage.2011.07.019

[4] Sha, L., Guo, H. and Song, A.W. (2003) An Improved Gridding Method for Spiral MRI Using Nonuniform Fast Fourier Transform. Journal of Magnetic Resonance, 162, 250-258. http://dx.doi.org/10.1016/S1090-7807(03)00107-1

[5] Dardzinski, B.J., Sotak, C.H., Fisher, M., Hasegawa, Y., Li, L. and Minematsu, K. (1993) Apparent Diffusion Coefficient Mapping of Experimental Focal Cerebral Ischemia Using Diffusion-Weighted Echo-Planar Imaging. Magnetic Resonance in Medicine, 30, 318-325. http://dx.doi.org/10.1002/mrm.1910300307

[6] Bammer, R., Auer, M., Keeling, S.L., Augustin, M., Stables, L.A., Prokesch, R.W., Stollberger, R., Moseley, M.E. and Fazekas, F. (2002) Diffusion Tensor Imaging Using Single-Shot SENSE-EPI. Magnetic Resonance in Medicine, 48, 128-136. http://dx.doi.org/10.1002/mrm.10184

[7] Moseley, M.E., Cohen, Y., Mintorovitch, J., Chileuitt, L., Shimizu, H., Kucharczyk, J., Wendland, M.F., Weinstein, P.R. and Fazekas, F. (1990) Early Detection of Regional Cerebral Ischemia in Cats: Comparison of Diffusion- and T2-Weighted MRI and Spectroscopy. Magnetic Resonance in Medicine, 14, 330-346. http://dx.doi.org/10.1002/mrm.1910140218

[8] Clark, C.A., Hedehus, M. and Moseley, M.E. (2001) Diffusion Time Dependence of the Apparent Diffusion Tensor in Healthy Human Brain and White Matter Disease. Magnetic Resonance in Medicine, 45, 1126-1129. http://dx.doi.org/10.1002/mrm.1149

[9] Zacharopoulos, N.G. and Narayana, P.A. (1998) Selective Measurement of White Matter and Gray Matter Diffusion Trace Values in Normal Human Brain. Medical Physics, 25, 2237-2241. http://dx.doi.org/10.1118/1.598424

[10] Basser, P.J., Mattiello, J. and LeBihan, D. (1994) Estimation of the Effective Self-Diffusion Tensor from the NMR Spin Echo. Journal of Magnetic Resonance, Series B, 103, 247-254. http://dx.doi.org/10.1006/jmrb.1994.1037

[11] Basser, P.J., Pajevic, S., Pierpaoli, C., Duda, J. and Aldroubi, A. (2000) In Vivo fiber Tractography Using DT-MRI Data. Magnetic Resonance in Medicine, 44, 625-632. http://dx.doi.org/10.1002/1522-2594(200010)44:4<625::AID-MRM17>3.0.CO;2-O

[12] Pierpaoli, C., Jezzard, P., Basser, P.J., Barnett, A. and Di Chiro, G. (1996) Diffusion Tensor MR Imaging of the Human Brain. Radiology, 201, 637-648.

[13] Frank, L.R., Jung, Y., Inati, S., Tyszka, J.M. and Wong, E.C. (2010) High Efficiency, Low Distortion 3D Diffusion Tensor Imaging with Variable Density Spiral Fast Spin Echoes (3D DW VDS RARE). NeuroImage, 49, 1510-1523. http://dx.doi.org/10.1016/j.neuroimage.2009.09.010

[14] Karampinos, D.C., Van, A.T., Olivero, W.C., Georgiadis, J.G. and Sutton, B.P. (2009) High-Resolution Diffusion 
Tensor Imaging of the Human Pons with a Reduced Field-of-View, Multishot, Variable-Density, Spiral Acquisition at 3 T. Magnetic Resonance in Medicine, 62, 1007-1016. http://dx.doi.org/10.1002/mrm.22105

[15] Liu, C., Bammer, R., Kim, D.H. and Moseley, M.E. (2004) Self-Navigated Interleaved Spiral (SNAILS): Application to High-Resolution Diffusion Tensor Imaging. Magnetic Resonance in Medicine, 52, 1388-1396. http://dx.doi.org/10.1002/mrm.20288

[16] Li, T.Q., Takahashi, A.M., Hindmarsh, T. and Moseley, M.E. (1990) ADC Mapping by Means of a Single-Shot Spiral MRI Technique with Application in Acute Cerebral Ischemia. Magnetic Resonance in Medicine, 41, 143-147. http://dx.doi.org/10.1002/(SICI)1522-2594(199901)41:1<143::AID-MRM20>3.0.CO;2-O

[17] Duyn, J.H. and Yang, Y. (1997) Fast Spiral Magnetic Resonance Imaging with Trapezoidal Gradients. Journal of Magnetic Resonance, 128, 130-134. http://dx.doi.org/10.1006/jmre.1997.1237

[18] Ellingson, B.M., Sulaiman, O. and Kurpad, S.N. (2010) High-Resolution in Vivo Diffusion Tensor Imaging of the Injured Cat Spinal Cord Using Self-Navigated, Interleaved, Variable-Density Spiral Acquisition (SNAILS-DTI). Magnetic Resonance Imaging, 28, 1353-1360. http://dx.doi.org/10.1016/j.mri.2010.06.006

[19] Voiron, J. and Lamalle, L. (2006) Spiral MRI: Principles and in Vivo Applications at High Field. In: Gerd, W. and William, E.H., Eds., Bruker Spin Report, Bruker BioSpin GmbH, 157-158, 9-17.

[20] Truong, T.K. and Guidon, A. (2014) High-Resolution Multishot Spiral Diffusion Tensor Imaging with Inherent Correction of Motion-Induced Phase Errors. Magnetic Resonance in Medicine, 71, 790-796.

[21] Holland, D.J., Liu, C., Song, X., Mazerolle, E.L., Stevens, M.T., Sederman, A.J., Gladden, L.F., D’Arcy, R.C., Bowen, C.V. and Beyea, S.D. (2013) Compressed Sensing Reconstruction Improves Sensitivity of Variable Density Spiral fMRI. Magnetic Resonance in Medicine, 70, 1634-1643. http://dx.doi.org/10.1002/mrm.24621 